A N N A LES

UNIVERSITATIS MARIAE CURIE-SKŁODOWSKA

L UBLIN - P OLONIA

VOL. LXVIII, NO. 2, 2014

SECTIO A

$27-50$

\title{
THOMAS ERNST
}

\section{On certain generalized $q$-Appell polynomial expansions}

\begin{abstract}
We study $q$-analogues of three Appell polynomials, the H-polynomials, the Apostol-Bernoulli and Apostol-Euler polynomials, whereby two new $q$-difference operators and the NOVA $q$-addition play key roles. The definitions of the new polynomials are by the generating function; like in our book, two forms, NWA and JHC are always given together with tables, symmetry relations and recurrence formulas. It is shown that the complementary argument theorems can be extended to the new polynomials as well as to some related polynomials. In order to find a certain formula, we introduce a $q$-logarithm. We conclude with a brief discussion of multiple $q$-Appell polynomials.
\end{abstract}

1. Introduction. The aim of this paper is to describe how the $q$-umbral calculus extends in a natural way to produce $q$-analogues of conversion theorems and polynomial expansions for the following Appell polynomials: $\mathrm{H}-$ polynomials, Apostol-Bernoulli and Apostol-Euler from the recent articles on this theme, as well as multiple variable extensions. To this aim, we use certain $q$-difference operators known from the book [3], and some new operators containing a factor $\lambda$ from the previous work of Luo and Srivastava [8], [9], [10] on Apostol-Bernoulli polynomials. The $q$-Appell polynomials have been used before in [4], where their basic definition was given together with

2010 Mathematics Subject Classification. Primary 05A40; 11B68; Secondary 05A10.

Key words and phrases. $q$-Apostol-Bernoulli polynomials, $q$-Apostol-Euler polynomials, $q$-H-polynomials, complementary argument theorem, generating function, multiple $q$-Appell polynomials. 
several matrix applications. The $q$-umbral method [3], influenced by Jordan [5] and Nørlund [12], forms the basis for the terminology and umbral method, which enables convenient $q$-analogues of the formulas for Appell polynomials; our formulas resemble the Appell polynomial formulas in a remarkable way. A certain $q$-Taylor formula plays a key role in many proofs.

This paper is organized as follows: In this section we give the general definitions. In each section, we then give the specific definitions and special values which we use there. In Section 2, we introduce two dual polynomials together with recursion formulas, symmetry relations and complementary argument theorem.

Let $\lambda \in \mathbb{R}$ and let $\mathrm{E}_{q}(x)$ denote the $q$-exponential function. In Sections 3 and 4 in the spirit of Apostol, Luo and Srivastava, we introduce and discuss two dual forms of the generalized $q$-Apostol-Bernoulli polynomials, together with the many applications that were mentioned earlier. In Sections 5 and 6 , we continue the discussion with two dual forms of the generalized $q$-ApostolEuler polynomials Two of their generating functions are given below:

$$
\frac{t^{n}}{\left(\lambda \mathrm{E}_{q}(t)-1\right)^{n}} \mathrm{E}_{q}(x t)=\sum_{\nu=0}^{\infty} \frac{t^{\nu} \mathcal{B}_{\mathrm{NWA}, \lambda, \nu, q}^{(n)}(x)}{\{\nu\}_{q} !},
$$

and

$$
\frac{2^{n}}{\left(\lambda \mathrm{E}_{q}(t)+1\right)^{n}} \mathrm{E}_{q}(x t)=\sum_{\nu=0}^{\infty} \frac{t^{\nu} \mathcal{F}_{\mathrm{NWA}, \lambda, \nu, q}^{(n)}(x)}{\{\nu\}_{q} !} .
$$

This is followed by formulas which contain both kinds of these polynomials. Many of the formulas are proved by simple manipulations of the generating functions. In Section 7 we show that the many expansion formulas according to Nørlund can also be formulated for our polynomials. In Section 7 we extend the previous considerations to a more general form, named multiplicative $q$-Appell polynomials. More on this will come in a future paper. We now come to Section 9; in order to find $q$-analogues of the corresponding formulas for the generating functions, we, formally, introduce a logarithm for the $q$-exponential function. The calculations are valid for so-called $q$-real numbers. In Section 10, we briefly discuss multiple $q$-Appell polynomials.

We now start with the definitions, compare with the book [3]. Some of the notation is well known and will be skipped.

Definition 1. Let the Gauss $q$-binomial coefficient be defined by

$$
\left(\begin{array}{l}
n \\
k
\end{array}\right)_{q} \equiv \frac{\{n\}_{q} !}{\{k\}_{q} !\{n-k\}_{q} !}, k=0,1, \ldots, n .
$$


Let $a$ and $b$ be any elements with commutative multiplication. Then the NWA $q$-addition is given by

$$
\left(a \oplus_{q} b\right)^{n} \equiv \sum_{k=0}^{n}\left(\begin{array}{l}
n \\
k
\end{array}\right)_{q} a^{k} b^{n-k}, n=0,1,2, \ldots
$$

The JHC $q$-addition is the function

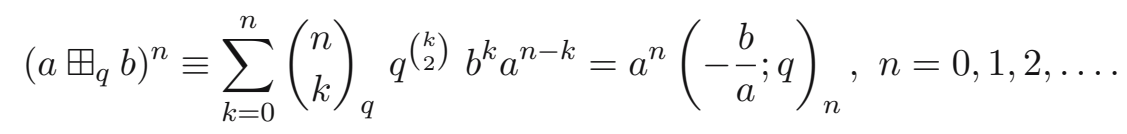

If $0<|q|<1$ and $|z|<|1-q|^{-1}$, the $q$-exponential function is defined by

$$
\mathrm{E}_{q}(z) \equiv \sum_{k=0}^{\infty} \frac{1}{\{k\}_{q} !} z^{k}
$$

The $q$-derivative is defined by

$$
\left(\mathrm{D}_{q} f\right)(x) \equiv \begin{cases}\frac{f(x)-f(q x)}{(1-q) x}, & \text { if } q \in \mathbb{C} \backslash\{1\}, x \neq 0 ; \\ \frac{d f}{d x}(x) & \text { if } q=1 ; \\ \frac{d f}{d x}(0) & \text { if } x=0 .\end{cases}
$$

Definition 2. Let the NWA $q$-shift operator be given by

$$
\mathrm{E}\left(\oplus_{q}\right)\left(x^{n}\right) \equiv\left(x \oplus_{q} 1\right)^{n} .
$$

Definition 3. The related JHC $q$-shift operator is given by

$$
\mathrm{E}\left(\boxplus_{q}\right)\left(x^{n}\right) \equiv\left(x \boxplus_{q} 1\right)^{n} .
$$

Let I denote the identity operator. The Apostol NWA $q$-difference operator is given by

$$
\triangle_{\mathrm{NWA}, \mathcal{A}, q} \equiv \lambda \mathrm{E}\left(\oplus_{q}\right)-\mathrm{I} .
$$

The Apostol NWA $q$-mean value operator is given by

$$
\nabla_{\mathrm{NWA}, \mathcal{A}, q} \equiv \frac{\lambda E\left(\oplus_{q}\right)+\mathrm{I}}{2} .
$$

The Apostol JHC $q$-difference operator is given by

$$
\triangle_{\mathrm{JHC}, \mathcal{A}, q} \equiv \lambda \mathrm{E}\left(\boxplus_{q}\right)-\mathrm{I} .
$$

The Apostol JHC $q$-mean value operator is given by

$$
\nabla_{\mathrm{JHC}, \mathcal{A}, q} \equiv \frac{\lambda E\left(\boxplus_{q}\right)+\mathrm{I}}{2} .
$$

Definition 4. For every power series $f_{n}(t)$, the $q$-Appell polynomials or $\Phi_{q}$ polynomials of degree $\nu$ and order $n$ have the following generating function:

$$
f_{n}(t) \mathrm{E}_{q}(x t)=\sum_{\nu=0}^{\infty} \frac{t^{\nu}}{\{\nu\}_{q} !} \Phi_{\nu, q}^{(n)}(x)
$$


For $x=0$ we get the $\Phi_{\nu, q}^{(n)}$ number of degree $\nu$ and order $n$.

Definition 5. For $f_{n}(t)$ of the form $h(t)^{n}$, we call the $q$-Appell polynomial $\Phi_{q}$ in (14) multiplicative.

Theorem 1.1. We have the two q-Taylor formulas

$$
\begin{gathered}
\Phi_{\nu, q}^{(n)}\left(x \oplus_{q} y\right)=\sum_{k=0}^{\nu}\left(\begin{array}{l}
\nu \\
k
\end{array}\right)_{q} \Phi_{\nu-k, q}^{(n)}(x) y^{k} . \\
\Phi_{\nu, q}^{(n)}\left(x \boxplus_{q} y\right)=\sum_{k=0}^{\nu}\left(\begin{array}{l}
\nu \\
k
\end{array}\right)_{q} q^{\left(\begin{array}{c}
k \\
2
\end{array}\right)} \Phi_{\nu-k, q}^{(n)}(x) y^{k} .
\end{gathered}
$$

2. The $\mathbf{H}$ polynomials. First, we repeat some of the definitions of certain related $q$-Appell polynomials for later use. These polynomials are more general forms of the polynomials we wish to study.

Definition 6. The generating function for $\beta_{\nu, q}^{(n)}(x)$ is

$$
\frac{t^{n} g(t)}{\left(\mathrm{E}_{q}(t)-1\right)^{n}} \mathrm{E}_{q}(x t) \equiv \sum_{\nu=0}^{\infty} \frac{t^{\nu} \beta_{\nu, q}^{(n)}(x)}{\{\nu\}_{q} !} .
$$

Definition 7. The generating function for $\gamma_{\nu, q}^{(n)}(x)$ is given by

$$
\frac{t^{n} g(t)}{\left(\mathrm{E}_{\bar{q}}(t)-1\right)^{n}} \mathrm{E}_{q}(x t) \equiv \sum_{\nu=0}^{\infty} \frac{t^{\nu} \gamma_{\nu, q}^{(n)}(x)}{\{\nu\}_{q} !} .
$$

Definition 8. The generating function for $\eta_{\nu, q}^{(n)}(x)$ is given by

$$
\frac{2^{n}}{\left(\mathrm{E}_{q}(t)+1\right)^{n}} g(t) \mathrm{E}_{q}(x t)=\sum_{\nu=0}^{\infty} \frac{t^{\nu} \eta_{\nu, q}^{(n)}(x)}{\{\nu\}_{q} !} .
$$

Definition 9. The generating function for $\theta_{\nu, q}^{(n)}(x)$ is given by

$$
\frac{2^{n}}{\left(\mathrm{E}_{\frac{1}{q}}(t)+1\right)^{n}} g(t) \mathrm{E}_{q}(x t)=\sum_{\nu=0}^{\infty} \frac{t^{\nu} \theta_{\nu, q}^{(n)}(x)}{\{\nu\}_{q} !} .
$$

We now come to the generating function of the polynomials we want to study in this section (for $q=1$ ): The $\mathrm{H}$ polynomials are defined in $[14, \mathrm{p}$. $532(37)]$.

$$
\frac{2 t}{e^{t}+1} e^{x t}=\sum_{\nu=0}^{\infty} \frac{t^{\nu} \mathrm{H}_{\nu}(x)}{\nu !}
$$

The odd $\mathrm{H}$ numbers are zero and the even $\mathrm{H}$ numbers are expressable in terms of Bernoulli numbers as

$$
\mathrm{H}_{2 n}=2\left(1-2^{2 n}\right) \mathrm{B}_{2 n} .
$$


Definition 10. The generating function for $\mathrm{H}_{\mathrm{NWA}, \nu, q}^{(n)}(x)$ is a special case of (19):

$$
\frac{(2 t)^{n}}{\left(\mathrm{E}_{q}(t)+1\right)^{n}} \mathrm{E}_{q}(x t)=\sum_{\nu=0}^{\infty} \frac{t^{\nu} \mathrm{H}_{\mathrm{NWA}, \nu, q}^{(n)}(x)}{\{\nu\}_{q} !},|t|<2 \pi .
$$

Definition 11. The generating function for $\mathrm{H}_{\mathrm{JHC}, \nu, q}^{(n)}(x)$ is a special case of (20):

$$
\frac{(2 t)^{n}}{\left(\mathrm{E}_{\frac{1}{q}}(t)+1\right)^{n}} \mathrm{E}_{q}(x t)=\sum_{\nu=0}^{\infty} \frac{t^{\nu} \mathrm{H}_{\mathrm{JHC}, \nu, q}^{(n)}(x)}{\{\nu\}_{q} !},|t|<2 \pi .
$$

The polynomials in (23) and (24) are $q$-analogues of the generalized $\mathrm{H}$ polynomials. We now turn to these $q$-analogues.

Theorem 2.1. We have

$$
\nabla_{\mathrm{NWA}, q} \mathrm{H}_{\mathrm{NWA}, \nu, q}^{(n)}(x)=\{\nu\}_{q} \mathrm{H}_{N W A, \nu-1, q}^{(n-1)}(x),
$$

(26) $\mathrm{H}_{\mathrm{NWA}, 0, q}=0, \mathrm{H}_{\mathrm{NWA}, 1, q}=1,\left(\mathrm{H}_{\mathrm{NWA}, q} \oplus_{q} 1\right)^{k}+\mathrm{H}_{\mathrm{NWA}, k, q} \ddot{=} 0, k>1$.

$$
\nabla_{\mathrm{JHC}, q} \mathrm{H}_{\mathrm{JHC}, \nu, q}^{(n)}(x)=\{\nu\}_{q} \mathrm{H}_{\mathrm{JHC}, \nu,-1, q}^{(n-1)}(x),
$$

$$
\mathrm{H}_{\mathrm{JHC}, 0, q}=0, \mathrm{H}_{\mathrm{JHC}, 1, q}=1,\left(\mathrm{H}_{\mathrm{JHC}, q} \boxplus_{q} 1\right)^{k}+\mathrm{H}_{\mathrm{JHC}, k, q} \ddot{=} 0, k>1 .
$$

The following table lists some of the first $\mathrm{H}_{\mathrm{NWA}, \nu, q}$ numbers.

$$
\begin{aligned}
& \begin{array}{|c|c|c|}
\hline n=2 & n=3 & n=4 \\
\hline(-1-q) 2^{-1} & \left(1-q^{3}\right) 2^{-2} & -(1+q)^{2}\left(1+q^{2}\right)\left(1-3 q+q^{2}\right) 2^{-3} \\
\hline
\end{array} \\
& \begin{array}{|c|}
\hline n=5 \\
\hline(-1+q)(1+q)\left(1-4 q+q^{2}\right)\{3\}_{q}\{5\}_{q} 2^{-4} \\
\hline
\end{array}
\end{aligned}
$$

We need not calculate the $\mathrm{H}_{\mathrm{JHC}, \nu,, q}$ numbers, since we have the following symmetry relations:

\section{Theorem 2.2.}

$$
\text { For } \nu \text { even, } \mathrm{H}_{\mathrm{NWA}, \nu, q}=\mathrm{H}_{\mathrm{JHC}, \nu, q} \text {. }
$$

For $\nu$ uneven, $\mathrm{H}_{\mathrm{NWA}, \nu, q}=-\mathrm{H}_{\mathrm{JHC}, \nu, q}, \nu>1$.

For the convenience of the reader, we make a short repetition:

Definition 12. The Ward $q$-Bernoulli numbers [17, p. 265, 16.4], are given by

$$
\mathrm{B}_{\mathrm{NWA}, n, q} \equiv \mathrm{B}_{\mathrm{NWA}, n, q}^{(1)} .
$$


The following table lists some of the first Ward $q$-Bernoulli numbers.

$$
\begin{array}{|c|c|c|c|}
\hline n=0 & n=1 & n=2 & n=3 \\
\hline 1 & -(1+q)^{-1} & q^{2}\left(\{3\}_{q} !\right)^{-1} & (1-q) q^{3}\left(\{2\}_{q}\right)^{-1}\left(\{4\}_{q}\right)^{-1} \\
\cline { 2 - 2 } & \multicolumn{3}{|c|}{n=4} \\
\cline { 2 - 3 } & \multicolumn{3}{|c|}{q^{4}\left(1-q^{2}-2 q^{3}-q^{4}+q^{6}\right)\left(\{2\}_{q}^{2}\{3\}_{q}\{5\}_{q}\right)^{-1}} \\
\cline { 2 - 3 }
\end{array}
$$

The following three formulas express $x^{n}$ in terms of $q$-Appell polynomials.

\section{Lemma 2.3.}

$$
x^{n}=\frac{1}{\{n+1\}_{q}} \sum_{k=0}^{n}\left(\begin{array}{c}
n+1 \\
k
\end{array}\right)_{q} \mathrm{~B}_{\mathrm{NWA}, k, q}(x)
$$

(see [3, 4.149, p. 121]).

$$
x^{\nu}=\frac{1}{2}\left[\mathrm{~F}_{\mathrm{NWA}, \nu, q}(x)+\sum_{k=0}^{\nu}\left(\begin{array}{l}
\nu \\
k
\end{array}\right)_{q} \mathrm{~F}_{\mathrm{NWA}, k, q}(x)\right]
$$

(see [3, 4.206, p. 130]).

$$
x^{\nu}=\frac{1}{2\{\nu+1\}_{q}}\left[\mathrm{H}_{\mathrm{NWA}, \nu+1, q}(x)+\sum_{k=0}^{\nu}\left(\begin{array}{l}
\nu+1 \\
k+1
\end{array}\right)_{q} \mathrm{H}_{\mathrm{NWA}, k+1, q}(x)\right] .
$$

Proof. For the last formula, use formula (25).

Theorem 2.4 (A q-analogue of [13, p. 489]). Let $\Phi_{\nu, q}^{(n)}(x)$ be a q-Appell polynomial. Then the following three addition formulas apply:

$$
\begin{gathered}
\Phi_{\nu, q}^{(n)}\left(x \oplus_{q} y\right) \\
=\sum_{k=0}^{\nu}\left[\sum_{j=k}^{\nu} \frac{1}{\{j+1\}_{q}}\left(\begin{array}{c}
\nu \\
j
\end{array}\right)_{q}\left(\begin{array}{c}
j+1 \\
k
\end{array}\right)_{q} \Phi_{\nu-j, q}^{(n)}(y)\right] \mathrm{B}_{\mathrm{NWA}, k, q}(x) . \\
\Phi_{\nu, q}^{(n)}\left(x \oplus_{q} y\right)=\frac{1}{2} \sum_{k=0}^{\nu}\left(\begin{array}{l}
\nu \\
k
\end{array}\right)_{q} \Phi_{\nu-k, q}^{(n)}(y) \\
\times\left[\mathrm{F}_{\mathrm{NWA}, k, q}(x)+\sum_{j=0}^{k}\left(\begin{array}{l}
k \\
j
\end{array}\right)_{q} \mathrm{~F}_{\mathrm{NWA}, j, q}(x)\right] . \\
\Phi_{\nu, q}^{(n)}\left(x \oplus_{q} y\right)=\frac{1}{2} \sum_{k=0}^{\nu}\left(\begin{array}{l}
\nu \\
k
\end{array}\right) \Phi_{q}^{(n)}\left(\begin{array}{l}
(n), \\
q
\end{array}\right) \frac{1}{\{k+1\}_{q}} \\
\times\left[\mathrm{H}_{\mathrm{NWA}, k+1, q}(x)+\sum_{j=0}^{k}\left(\begin{array}{l}
k+1 \\
j+1
\end{array}\right)_{q} \mathrm{H}_{\mathrm{NWA}, j+1, q}(x)\right] .
\end{gathered}
$$


Proof. Use formulas (31)-(33) and (15).

The following complementary argument theorems extend the ones given in [3, p. 153].

Theorem 2.5. A q-analogue of the Raabe-Bernoulli complementary argument theorem $[11$, p. 128, (1)]:

$$
\mathrm{B}_{\mathrm{JHC}, \nu, q}^{(n)}(x)=(-1)^{\nu} \mathrm{B}_{\mathrm{NWA}, \nu, q}^{(n)}\left(\bar{n}_{q} \ominus_{q} x\right) .
$$

Theorem 2.6. A q-analogue of the Euler complementary argument theorem by Milne-Thomson [11, p. 145, (1)]:

$$
\mathrm{F}_{\mathrm{JHC}, \nu, q}^{(n)}(x)=(-1)^{\nu} \mathrm{F}_{\mathrm{NWA}, \nu, q}^{(n)}\left(\bar{n}_{q} \ominus_{q} x\right) .
$$

These two formulas can be generalized to

Theorem 2.7 (A q-analogue of the Raabe-Bernoulli complementary argument theorem $[11$, p. 128, (1)]). Assume that $g(t)$ in (17) and (18) are equal and even functions. Then

$$
\gamma_{\nu, q}^{(n)}(x)=(-1)^{\nu} \beta_{\nu, q}^{(n)}\left(\bar{n}_{q} \ominus_{q} x\right) .
$$

Theorem 2.8 (A q-analogue of the Euler complementary argument theorem by Milne-Thomson [11, p. 145, (1)]). Assume that $g(t)$ in (19) and (20) are equal and even functions. Then

$$
\theta_{\nu, q}^{(n)}(x)=(-1)^{\nu} \eta_{\nu, q}^{(n)}\left(\bar{n}_{q} \ominus_{q} x\right) .
$$

Theorem 2.9. A special case of (40), and a q-analogue of [14, p. 532]:

$$
\mathrm{H}_{\mathrm{JHC}, \nu, q}^{(n)}(x)=(-1)^{\nu+n} \mathrm{H}_{\mathrm{NWA}, \nu, q}^{(n)}\left(\left(\bar{n}_{q} \ominus_{q} x\right) .\right.
$$

Proof. Use the generating function.

Corollary 2.10. A q-analogue of a generalization of [14, p. 532].

$$
\mathrm{H}_{\mathrm{NWA}, \nu, q}^{(n)}(x)+(-1)^{\nu+n} \mathrm{H}_{\mathrm{JHC}, \nu, q}^{(n)}(-x)=2\{\nu\}_{q} \mathrm{H}_{\mathrm{NWA}, \nu-1, q}^{(n-1)}(x) .
$$

Proof. Use the generating function and (25), (41).

3. The NWA $q$-Apostol-Bernoulli polynomials. Throughout, we assume that $\lambda \neq 0$. The $\mathrm{b}$ polynomials are more general forms of the NWA $q$-Apostol-Bernoulli polynomials, which we will study in this section.

Definition 13. The polynomials $\mathrm{b}_{\lambda, \nu, q}^{(n)}(x)$ are defined by

$$
\frac{t^{n} g(t)}{\left(\lambda \mathrm{E}_{q}(t)-1\right)^{n}} \mathrm{E}_{q}(x t)=\sum_{\nu=0}^{\infty} \frac{t^{\nu} \mathrm{b}_{\lambda, \nu, q}^{(n)}(x)}{\{\nu\}_{q} !} .
$$

The generating function for $\mathrm{B}_{\mathrm{NWA}, \nu, q}(x)$ is a special case of (44): 
Definition 14. The generalized NWA $q$-Apostol-Bernoulli polynomials $\mathcal{B}_{\mathrm{NWA}, \lambda, \nu, q}^{(n)}(x)$ are defined by

$$
\frac{t^{n}}{\left(\lambda \mathrm{E}_{q}(t)-1\right)^{n}} \mathrm{E}_{q}(x t)=\sum_{\nu=0}^{\infty} \frac{t^{\nu} \mathcal{B}_{\mathrm{NWA}, \lambda, \nu, q}^{(n)}(x)}{\{\nu\}_{q} !},|t+\log \lambda|<2 \pi .
$$

Assume that $\lambda \neq 1$. The poles in the denominator of (44) are the roots of $\mathrm{E}_{q}(t)=\lambda^{-1}$, which implies that in some cases the limit $\lambda \rightarrow 1$ is not straightforward and needs some further consideration.

We have

$$
\triangle_{\mathrm{NWA}, \mathcal{A}, q} \mathrm{~b}_{\lambda, \nu, q}^{(n)}(x)=\{\nu\}_{q} \mathrm{~b}_{\lambda, \nu-1, q}^{(n-1)}(x)=\mathrm{D}_{q} \mathrm{~b}_{\lambda, \nu, q}^{(n-1)}(x) .
$$

This leads to the following recurrence for the NWA $q$-Apostol-Bernoulli numbers:

$$
\mathcal{B}_{\mathrm{NWA}, \lambda, 0, q}=0, \lambda\left(\mathcal{B}_{\mathrm{NWA}, \lambda, q} \oplus_{q} 1\right)^{k}-\mathcal{B}_{\mathrm{NWA}, \lambda, k, q} \ddot{=} \delta_{1, k}, k>0 .
$$

The following table lists some of the first $\mathcal{B}_{\mathrm{NWA}, \lambda, \nu, q}$ numbers.

$$
\begin{array}{|c|c|c|c|}
\hline \nu=0 & \nu=1 & \nu=2 & \nu=3 \\
\hline 0 & \frac{1}{1-\lambda} & -\frac{\lambda(1+q)}{(1-\lambda)^{2}} & \frac{\lambda(1+\lambda q)\{3\}_{q}}{(\lambda-1)^{3}} \\
\hline \multicolumn{3}{|c|}{\nu=4} & \nu=5 \\
\hline-\frac{\lambda\{2\}_{q}\left(1+q^{2}\right)\left(1+2 \lambda q+2 \lambda q^{2}+\lambda^{2} q^{3}\right)}{(1-\lambda)^{4}} & \frac{A \lambda\{5\}_{q}}{(\lambda-1)^{5}} \\
\hline
\end{array}
$$

where

(47) $A \equiv 1+3 \lambda q+4 \lambda q^{2}+\lambda^{3} q^{6}+\lambda q^{3}(3+q)+\lambda^{2} q^{2}\left(1+3 q+4 q^{2}+3 q^{3}\right)$.

Theorem 3.1 (A generalization of $[3,4.242]$ ). If $\sum_{l=1}^{s} n_{l}=n$,

$$
\begin{aligned}
& \mathrm{B}_{\mathrm{NWA}, \lambda, k, q}^{(n)}\left(x_{1} \oplus_{q} \ldots \oplus_{q} x_{s}\right) \\
& \quad=\sum_{m_{1}+\ldots+m_{s}=k}\left(\begin{array}{c}
k \\
m_{1}, \ldots, m_{s}
\end{array}\right) \prod_{q}^{s} \mathrm{~B}_{\mathrm{NWA}, \lambda, m_{j}, q}^{\left(n_{j}\right)}\left(x_{j}\right),
\end{aligned}
$$

where we assume that $n_{j}$ operates on $x_{j}$.

Corollary 3.2 (A q-analogue of [8, p. 300 (49)]).

$$
\mathcal{B}_{\mathrm{NWA}, \lambda, \nu, q}^{(n)}(x)=\sum_{k=0}^{\nu}\left(\begin{array}{l}
\nu \\
k
\end{array}\right)_{q} \mathcal{B}_{\mathrm{NWA}, \lambda, \nu-k, q}^{(n-1)} \mathcal{B}_{\mathrm{NWA}, \lambda, k, q}(x) .
$$

Corollary 3.3. A q-analogue of [9, p. 634, (28), (29)]:

$$
\begin{aligned}
& \mathcal{B}_{\mathrm{NWA}, \lambda, \nu, q}^{(n-1)}(x) \\
& \quad=\frac{1}{\{\nu+1\}_{q}}\left[\lambda \sum_{k=0}^{\nu+1}\left(\begin{array}{c}
\nu+1 \\
k
\end{array}\right)_{q} \mathcal{B}_{\mathrm{NWA}, \lambda, k, q}^{(n)}(x)-\mathcal{B}_{\mathrm{NWA}, \lambda, \nu+1, q}^{(n)}(x)\right] .
\end{aligned}
$$


A generalization of $[3,4.149]$.

$$
x^{\nu}=\frac{1}{\{\nu+1\}_{q}}\left[\lambda \sum_{k=0}^{\nu+1}\left(\begin{array}{c}
\nu+1 \\
k
\end{array}\right)_{q} \mathcal{B}_{\mathrm{NWA}, \lambda, k, q}(x)-\mathcal{B}_{\mathrm{NWA}, \lambda, \nu+1, q}(x)\right] .
$$

4. The JHC q-Apostol-Bernoulli polynomials. The c polynomials are more general forms of the JHC q-Apostol-Bernoulli polynomials, which we will study in this section.

Definition 15. The polynomials $c_{\lambda, \nu, q}^{(n)}(x)$ are defined by

$$
\frac{t^{n} g(t)}{\left(\lambda \mathrm{E}_{\frac{1}{q}}(t)-1\right)^{n}} \mathrm{E}_{q}(x t)=\sum_{\nu=0}^{\infty} \frac{t^{\nu} \mathrm{c}_{\lambda, \nu, q}^{(n)}(x)}{\{\nu\}_{q} !} .
$$

Definition 16. The generalized JHC $q$-Apostol-Bernoulli polynomials $\mathcal{B}_{\mathrm{JHC}, \lambda, \nu, q}^{(n)}(x)$ are defined by

$$
\frac{t^{n}}{\left(\lambda \mathrm{E}_{\frac{1}{q}}(t)-1\right)^{n}} \mathrm{E}_{q}(x t)=\sum_{\nu=0}^{\infty} \frac{t^{\nu} \mathcal{B}_{\mathrm{JHC}, \lambda, \nu, q}^{(n)}(x)}{\{\nu\}_{q} !},|t+\log \lambda|<2 \pi .
$$

We have

$$
\triangle_{\mathrm{JHC}, \mathcal{A}, q} \mathrm{c}_{\lambda, \nu, q}^{(n)}(x)=\{\nu\}_{q} \mathrm{c}_{\lambda, \nu-1, q}^{(n-1)}(x)=\mathrm{D}_{q} \mathrm{c}_{\lambda, \nu, q}^{(n-1)}(x) .
$$

The defining relation of the JHC $q$-Apostol-Bernoulli numbers is given by the umbral recurrence

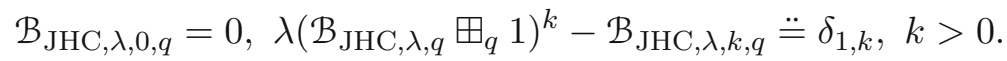

We need not calculate the $\mathcal{B}_{\mathrm{JHC}, \lambda, \nu, q}$ numbers, since we have the following symmetry relations:

Theorem 4.1. Assume that $g(t)$ in (43) and (52) are equal and even functions. Then

$$
\mathrm{b}_{\lambda, \nu, q}=(-1)^{\nu} \mathrm{c}_{\lambda^{-1}, \nu, q}, \nu>0 .
$$

In particular,

$$
\mathcal{B}_{\mathrm{NWA}, \lambda, \nu, q}=(-1)^{\nu} \mathcal{B}_{\mathrm{JHC}, \lambda^{-1}, \nu, q}, \nu>0 .
$$

Theorem 4.2. Assume that $g(t)$ in (43) and (52) are equal and even functions. Then

$$
\mathrm{c}_{\lambda^{-1}, \nu, q}^{(n)}(x)=(-1)^{\nu} \lambda^{n} \mathrm{~b}_{\lambda, \nu, q}^{(n)}\left(\bar{n}_{q} \ominus_{q} x\right) .
$$

This implies a $q$-analogue of the complementary argument theorem $[9$, p. 633, (19)].

\section{Theorem 4.3.}

$$
\mathcal{B}_{\mathrm{JHC}, \lambda^{-1}, \nu, q}^{(n)}(x)=(-1)^{\nu} \lambda^{n} \mathcal{B}_{\mathrm{NWA}, \lambda, \nu, q}^{(n)}\left(\bar{n}_{q} \ominus_{q} x\right) .
$$


Theorem 4.4 (Compare with $[3,4.242])$. If $\sum_{l=1}^{s} n_{l}=n$,

$$
\begin{aligned}
& \mathrm{B}_{\mathrm{JHC}, \lambda, k, q}^{(n)}\left(x_{1} \oplus_{q} \ldots \oplus_{q} x_{s}\right) \\
& \quad=\sum_{m_{1}+\ldots+m_{s}=k}\left(\begin{array}{c}
k \\
m_{1}, \ldots, m_{s}
\end{array}\right) \prod_{q}^{s} \mathrm{~B}_{\mathrm{JHC}, \lambda, m_{j}, q}^{\left(n_{j}\right)}\left(x_{j}\right),
\end{aligned}
$$

where we assume that $n_{j}$ operates on $x_{j}$.

Corollary 4.5 (Another q-analogue of [8, p. 300 (49)]).

$$
\mathcal{B}_{\mathrm{JHC}, \lambda, \nu, q}^{(n)}(x)=\sum_{k=0}^{\nu}\left(\begin{array}{l}
\nu \\
k
\end{array}\right)_{q} \mathcal{B}_{\mathrm{JHC}, \lambda, \nu-k, q}^{(n-1)} \mathcal{B}_{\mathrm{JHC}, \lambda, k, q}(x) .
$$

Corollary 4.6 (Another $q$-analogue of [9, p. 634, (28), (29)]).

$$
\begin{aligned}
& \mathcal{B}_{\mathrm{JHC}, \lambda, \nu, q}^{(n-1)}(x) \\
& =\frac{1}{\{\nu+1\}_{q}}\left[\lambda \sum_{k=0}^{\nu+1}\left(\begin{array}{c}
\nu+1 \\
k
\end{array}\right)_{q} q^{\left(\begin{array}{c}
k \\
2
\end{array}\right)} \mathcal{B}_{\mathrm{JHC}, \lambda, \nu+1-k, q}^{(n)}(x)\right. \\
& \left.\quad-\mathcal{B}_{\mathrm{JHC}, \lambda, \nu+1, q}^{(n)}(x)\right] .
\end{aligned}
$$

Theorem 4.7 (A q-analogue of $[6$, p. 8]).

$$
\begin{aligned}
& \sum_{k=0}^{\nu}\left(\begin{array}{l}
\nu \\
k
\end{array}\right)_{q} \mathcal{B}_{\mathrm{NWA}, \lambda, k, q}^{(2 n)}\left(x \ominus_{q} y\right)\left(\bar{n}_{q}\right)^{\nu-k} \\
& =\frac{1}{\lambda^{n}} \sum_{k=0}^{\nu}(-1)^{\nu-k}\left(\begin{array}{l}
\nu \\
k
\end{array}\right)_{q} \mathcal{B}_{\mathrm{NWA}, \lambda, k, q}^{(n)}(x) \mathcal{B}_{\mathrm{JHC}, \lambda^{-1}, \nu-k, q}^{(n)}(y) .
\end{aligned}
$$

Proof. We have that

$$
\begin{aligned}
& \frac{t^{n}}{\left(\lambda \mathrm{E}_{q}(t)-1\right)^{n}} \mathrm{E}_{q}(x t) \frac{(-t)^{n}}{\left(\lambda^{-1} \mathrm{E}_{\frac{1}{q}}(-t)-1\right)^{n}} \mathrm{E}_{q}(-y t) \\
& =\mathrm{E}_{q}\left(\left(x \ominus_{q} y\right) t\right) \frac{t^{n}}{\left(\lambda \mathrm{E}_{q}(t)-1\right)^{n}}\left(\frac{t \lambda \mathrm{E}_{q}(t)}{\lambda \mathrm{E}_{q}(t)-1}\right)^{n},
\end{aligned}
$$

which implies that

$$
\begin{aligned}
& \sum_{\nu=0}^{\infty} \frac{t^{\nu} \mathcal{B}_{\mathrm{NWA}, \lambda, \nu, q}^{(2 n)}\left(x \ominus_{q} y\right)}{\{\nu\}_{q} !} \sum_{l=0}^{\infty} \frac{\left(\bar{n}_{q} t\right)^{l}}{\{l\}_{q} !} \\
& =\frac{1}{\lambda^{n}} \sum_{\nu=0}^{\infty} \frac{t^{\nu} \mathcal{B}_{\mathrm{NWA}, \lambda, \nu, q}^{(n)}(x)}{\{\nu\}_{q} !} \sum_{m=0}^{\infty} \frac{(-t)^{m} \mathcal{B}_{\mathrm{JHC}^{\prime} \lambda^{-1}, m, q}^{(n)}(y)}{\{m\}_{q} !} .
\end{aligned}
$$

Formula (63) now follows on equating the coefficients of $t^{\nu}$. 
5. The NWA $\boldsymbol{q}$-Apostol-Euler polynomials. We start with some repetition from [3]:

Definition 17. The generating function for the first $q$-Euler polynomials of degree $\nu$ and order $n, \mathrm{~F}_{\mathrm{NWA}, \nu, q}^{(n)}(x)$ is given by

$$
\frac{2^{n} \mathrm{E}_{q}(x t)}{\left(\mathrm{E}_{q}(t)+1\right)^{n}}=\sum_{\nu=0}^{\infty} \frac{t^{\nu}}{\{\nu\}_{q} !} \mathrm{F}_{\mathrm{NWA}, \nu, q}^{(n)}(x),|t|<\pi .
$$

The following table lists some of the first $q$-Euler numbers $\mathrm{F}_{\mathrm{NWA}, n, q}$.

$$
\begin{aligned}
& \begin{array}{|c|c|c|c|}
\hline n=0 & n=1 & n=2 & n=3 \\
\hline 1 & -2^{-1} & 2^{-2}(-1+q) & 2^{-3}\left(-1+2 q+2 q^{2}-q^{3}\right) \\
\hline
\end{array} \\
& \begin{array}{|c|}
\hline n=4 \\
\hline 2^{-4}(q-1)\{3\}_{q} !\left(q^{2}-4 q+1\right) \\
\hline
\end{array}
\end{aligned}
$$

The e polynomials are more general forms of the NWA $q$-Apostol-Euler polynomials, which we will study in this section.

Definition 18. The e polynomials are defined by

$$
\frac{2^{n} g(t)}{\left(\lambda \mathrm{E}_{q}(t)+1\right)^{n}} \mathrm{E}_{q}(x t)=\sum_{\nu=0}^{\infty} \frac{t^{\nu} \mathrm{e}_{\lambda, \nu, q}^{(n)}(x)}{\{\nu\}_{q} !} .
$$

Definition 19. The generalized NWA $q$-Apostol-Euler polynomials $\mathcal{F}_{\text {NWA, }, \nu, q}^{(n)}(x)$ are defined by

$$
\frac{2^{n}}{\left(\lambda \mathrm{E}_{q}(t)+1\right)^{n}} \mathrm{E}_{q}(x t)=\sum_{\nu=0}^{\infty} \frac{t^{\nu} \mathcal{F}_{\mathrm{NWA}, \lambda, \nu, q}^{(n)}(x)}{\{\nu\}_{q} !},|t+\log \lambda|<\pi .
$$

Assume that $\lambda \neq-1$. The poles in the denominator of (68) are the roots of $\mathrm{E}_{q}(t)=-\lambda^{-1}$.

Theorem 5.1. We have

$$
\nabla_{\mathrm{NWA}, \mathcal{A}, q} \mathrm{e}_{\lambda, \nu, q}^{(n)}(x)=\mathrm{e}_{\lambda, \nu, q}^{(n-1)}(x),
$$

This leads to the following recurrence:

The following table lists some of the first $\mathcal{F}_{\mathrm{NWA}, \lambda, n, q}$ numbers.

\begin{tabular}{|c|c|c|c|}
\hline$n=0$ & $n=1$ & $n=2$ & $n=3$ \\
\hline$\frac{2}{1+\lambda}$ & $\frac{-2}{(1+\lambda)^{2}}$ & $\frac{2(q-\lambda)}{(1+\lambda)^{3}}$ & $\frac{-2\left(\lambda^{2}+q^{3}-2 \lambda q-2 \lambda q^{2}\right)}{(\lambda+1)^{4}}$ \\
\hline
\end{tabular}

We observe that the limits for $\lambda \rightarrow 1$ are the first $q$-Euler numbers. The following two formulas are generalizations of [3, 4.202] and [3, 4.206]. 
Theorem 5.2 (A q-analogue of [9, p. 635, (31), (32)]).

$$
\mathcal{F}_{\mathrm{NWA}, \lambda, \nu, q}^{(n-1)}(x)=\frac{1}{2}\left[\lambda \sum_{k=0}^{\nu}\left(\begin{array}{l}
\nu \\
k
\end{array}\right)_{q} \mathcal{F}_{\mathrm{NWA}, \lambda, k, q}^{(n)}(x)+\mathcal{F}_{\mathrm{NWA}, \lambda, \nu, q}^{(n)}(x)\right] .
$$

$$
x^{\nu}=\frac{1}{2}\left[\lambda \sum_{k=0}^{\nu}\left(\begin{array}{l}
\nu \\
k
\end{array}\right)_{q} \mathcal{F}_{\mathrm{NWA}, \lambda, k, q}(x)+\mathcal{F}_{\mathrm{NWA}, \lambda, \nu, q}(x)\right] .
$$

Theorem 5.3. A q-analogue of $[9$, p. $636,(43)]$ and a generalization of $[3$, p. 152]:

$$
\begin{aligned}
& \mathcal{B}_{\mathrm{NWA}, \lambda, \nu, q}^{(n)}\left(x \oplus_{q} y\right) \\
& =\sum_{k=0}^{\nu}\left(\begin{array}{l}
\nu \\
k
\end{array}\right)_{q}\left[\mathcal{B}_{\mathrm{NWA}, \lambda, k, q}^{(n)}(y)+\frac{\{k\}_{q}}{2} \mathcal{B}_{\mathrm{NWA}, \lambda, k-1, q}^{(n-1)}(y)\right] \mathcal{F}_{\mathrm{NWA}, \lambda, \nu-k, q}(x) .
\end{aligned}
$$

Proof. We will use the $q$-Taylor formula (15) twice, and then like in [9], the factor $\lambda$ disappears.

$$
\begin{aligned}
& \mathcal{B}_{\mathrm{NWA}, \lambda, \nu, q}^{(n)}\left(x \oplus_{q} y\right) \\
& \stackrel{\operatorname{by}(15,72)}{=} \frac{1}{2} \sum_{k=0}^{\nu}\left(\begin{array}{l}
\nu \\
k
\end{array}\right)_{q} \mathcal{B}_{\mathrm{NWA}, \lambda, k, q}^{(n)}(y)\left[\mathcal{F}_{\mathrm{NWA}, \lambda, \nu-k, q}(x)\right. \\
& \left.+\lambda \sum_{j=0}^{\nu-k}\left(\begin{array}{c}
\nu-k \\
j
\end{array}\right)_{q} \mathcal{F}_{\mathrm{NWA}, \lambda, j, q}(x)\right] \\
& =\frac{1}{2} \sum_{k=0}^{\nu}\left(\begin{array}{l}
\nu \\
k
\end{array}\right)_{q} \mathcal{B}_{\mathrm{NWA}, \lambda, k, q}^{(n)}(y) \mathcal{F}_{\mathrm{NWA}, \lambda, \nu-k, q}(x) \\
& +\frac{\lambda}{2} \sum_{j=0}^{\nu}\left(\begin{array}{l}
\nu \\
j
\end{array}\right)_{q} \mathcal{F}_{\mathrm{NWA}, \lambda, j, q}(x) \sum_{k=0}^{\nu-j}\left(\begin{array}{c}
\nu-j \\
k
\end{array}\right)_{q} \mathcal{B}_{\mathrm{NWA}, \lambda, k, q}^{(n)}(y)
\end{aligned}
$$

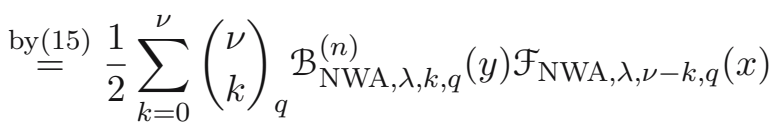

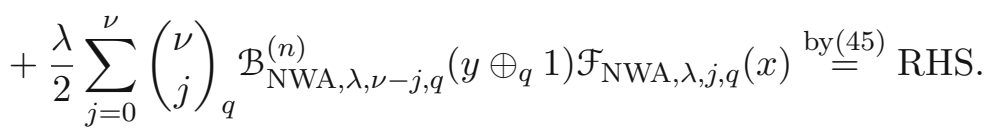


Theorem 5.4 (Another $q$-analogue of $[9$, p. 636, (43)]).

$$
\begin{gathered}
\mathcal{B}_{\mathrm{JHC}, \lambda, \nu, q}^{(n)}\left(x \boxplus_{q} y\right)=\sum_{k=0}^{\nu}\left(\begin{array}{l}
\nu \\
k
\end{array}\right)_{q}\left[\mathcal{B}_{\mathrm{JHC}, \lambda, k, q}^{(n)}(y)\left(\frac{q^{\left(\begin{array}{c}
k \\
2
\end{array}\right)}+1}{2}\right)\right. \\
\left.+q^{\left(\begin{array}{c}
k \\
2
\end{array}\right)} \frac{\{k\}_{q}}{2} \mathcal{B}_{\mathrm{JHC}, \lambda, k-1, q}^{(n-1)}(y)\right] \mathcal{F}_{\mathrm{NWA}, \lambda, \nu-k, q}(x) .
\end{gathered}
$$

Proof. We will use the $q$-Taylor formula (16) twice, and then like in [9], the factor $\lambda$ disappears.

$$
\begin{aligned}
& \mathcal{B}_{\mathrm{JHC}, \lambda, \nu, q}^{(n)}\left(x \boxplus_{q} y\right) \\
& \stackrel{\operatorname{by}(\underline{16,72)}}{=} \frac{1}{2} \sum_{k=0}^{\nu}\left(\begin{array}{l}
\nu \\
k
\end{array}\right)_{q} q^{\left(\begin{array}{l}
k \\
2
\end{array}\right)} \mathcal{B}_{\mathrm{JHC}, \lambda, k, q}^{(n)}(y)\left[\mathcal{F}_{\mathrm{NWA}, \lambda, \nu-k, q}(x)\right. \\
& \left.+\lambda \sum_{j=0}^{\nu-k}\left(\begin{array}{c}
\nu-k \\
j
\end{array}\right)_{q} \mathcal{F}_{\mathrm{NWA}, \lambda, j, q}(x)\right]
\end{aligned}
$$

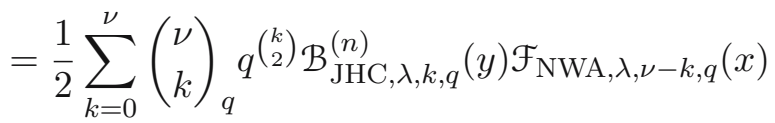

$$
\begin{aligned}
& +\frac{\lambda}{2} \sum_{j=0}^{\nu}\left(\begin{array}{l}
\nu \\
j
\end{array}\right)_{q} \mathcal{F}_{\mathrm{NWA}, \lambda, j, q}(x) \sum_{k=0}^{\nu-j}\left(\begin{array}{c}
\nu-j \\
k
\end{array}\right)_{q} q^{\left(\begin{array}{c}
k \\
2
\end{array}\right)} \mathcal{B}_{\mathrm{JHC}, \lambda, k, q}^{(n)}(y) \\
& \stackrel{\text { by }(16)}{=} \frac{1}{2} \sum_{k=0}^{\nu}\left(\begin{array}{l}
\nu \\
k
\end{array}\right)_{q} q^{\left(\begin{array}{l}
k \\
2
\end{array}\right)} \mathcal{B}_{\mathrm{JHC}, \lambda, k, q}^{(n)}(y) \mathcal{F}_{\mathrm{NWA}, \lambda, \nu-k, q}(x) \\
& +\frac{\lambda}{2} \sum_{j=0}^{\nu}\left(\begin{array}{l}
\nu \\
j
\end{array}\right)_{q} \mathcal{B}_{\mathrm{JHC}, \lambda, \nu-j, q}^{(n)}\left(y \boxplus_{q} 1\right) \mathcal{F}_{\mathrm{NWA}, \lambda, j, q}(x) \stackrel{\text { by } \stackrel{(54)}{=}}{\mathrm{RHS} .}
\end{aligned}
$$

Theorem 5.5 (A $q$-analogue of the addition theorem [6, p. 10]).

$$
\begin{aligned}
& \mathcal{B}_{\mathrm{NWA}, \lambda^{2}, \nu, q}^{(n)}\left(x \oplus_{q} y\right) \\
& =\frac{\left(\overline{2}_{q}\right)^{n}}{2^{n}\left(\overline{2}_{q}\right)^{\nu}} \sum_{k=0}^{\nu}\left(\begin{array}{l}
\nu \\
k
\end{array}\right)_{q} \mathcal{B}_{\mathrm{NWA}, \lambda, k, q}^{(n)}\left(\overline{2}_{q} x\right) \mathcal{F}_{\mathrm{NWA}, \lambda, \nu-k, q}^{(n)}\left(\overline{2}_{q} y\right) .
\end{aligned}
$$

Proof. We find that

$$
\begin{aligned}
& \frac{2^{n}}{\left(\overline{2}_{q}\right)^{n}}\left(\frac{\overline{2}_{q} t}{\lambda^{2} \mathrm{E}_{q}\left(\overline{2}_{q} t\right)-1}\right)^{n} \mathrm{E}_{q}\left(\left(\overline{2}_{q} x \oplus_{q} \overline{2}_{q} y\right) t\right) \\
& =\left(\frac{t}{\left(\lambda \mathrm{E}_{q}(t)-1\right.}\right)^{n} \mathrm{E}_{q}\left(\overline{2}_{q} x t\right) \frac{2^{n}}{\left(\lambda \mathrm{E}_{q}(t)+1\right)^{n}} \mathrm{E}_{q}\left(\overline{2}_{q} y t\right),
\end{aligned}
$$


which implies that

$$
\begin{aligned}
& \frac{2^{n}}{\left(\overline{2}_{q}\right)^{n}} \sum_{\nu=0}^{\infty} \frac{\left(\overline{2}_{q} t\right)^{\nu} \mathcal{B}_{\mathrm{NWA}, \lambda^{2}, \nu, q}^{(n)}\left(x \oplus_{q} y\right)}{\{\nu\}_{q} !} \\
& =\sum_{\nu=0}^{\infty} \frac{t^{\nu} \mathcal{B}_{\mathrm{NWA}, \lambda, \nu, q}^{(n)}\left(\overline{2}_{q} x\right)}{\{\nu\}_{q} !} \sum_{m=0}^{\infty} \frac{t^{m} \mathcal{F}_{\mathrm{NWA}, \lambda, m, q}^{(n)}\left(\overline{2}_{q} y\right)}{\{m\}_{q} !} .
\end{aligned}
$$

Formula (77) now follows on equating the coefficients of $t^{\nu}$.

6. The JHC $q$-Apostol-Euler polynomials. The $f$ polynomials are more general forms of the JHC q-Apostol-Euler polynomials, which we will study in this section.

Definition 20. The f polynomials $\mathrm{f}_{\lambda, \nu, q}^{(n)}(x)$ are defined by

$$
\frac{2^{n} g(t)}{\left(\lambda \mathrm{E}_{\frac{1}{q}}(t)+1\right)^{n}} \mathrm{E}_{q}(x t)=\sum_{\nu=0}^{\infty} \frac{t^{\nu} \mathrm{f}_{\lambda, \nu, q}^{(n)}(x)}{\{\nu\}_{q} !} .
$$

Definition 21. The generalized JHC $q$-Apostol-Euler polynomials $\mathcal{F}_{\mathrm{JHC}, \lambda, \nu, q}^{(n)}(x)$ are defined by

$$
\frac{2^{n}}{\left(\lambda \mathrm{E}_{\frac{1}{q}}(t)+1\right)^{n}} \mathrm{E}_{q}(x t)=\sum_{\nu=0}^{\infty} \frac{t^{\nu} \mathcal{F}_{\mathrm{JHC}, \lambda, \nu, q}^{(n)}(x)}{\{\nu\}_{q} !},|t+\log \lambda|<\pi .
$$

Theorem 6.1. We have

$$
\nabla_{\mathrm{JHC}, \mathcal{A}, q} \mathrm{f}_{\lambda, \nu, q}^{(n)}(x)=\mathrm{f}_{\lambda, \nu, q}^{(n-1)}(x),
$$

This leads to the following recurrence:

$$
\mathcal{F}_{\mathrm{JHC}, \lambda, 0, q}=2-\lambda, \quad \lambda\left(\mathcal{F}_{\mathrm{JHC}, \lambda, q} \boxplus_{q} 1\right)^{k}+\mathcal{F}_{\mathrm{JHC}, \lambda, k, q} \ddot{=} 0, k>1 .
$$

Theorem 6.2. A generalization of $[3,4.224]$ and another q-analogue of $[9$, p. $635,(31)]$ :

$$
\mathcal{F}_{\mathrm{JHC}, \lambda, \nu, q}^{(n-1)}(x)=\frac{1}{2}\left[\lambda \sum_{k=0}^{\nu}\left(\begin{array}{l}
\nu \\
k
\end{array}\right)_{q} q^{\left(\begin{array}{l}
k \\
2
\end{array}\right)} \mathcal{F}_{\mathrm{JHC}, \lambda, \nu-k, q}^{(n)}(x)+\mathcal{F}_{\mathrm{JHC}, \lambda, \nu, q}^{(n)}(x)\right] .
$$

Theorem 6.3. A symmetry relation for $q$-Apostol-Euler numbers.

$$
(-1)^{\nu} \mathcal{F}_{\mathrm{JHC}, \lambda^{-1}, \nu, q}=-\mathcal{F}_{\mathrm{NWA}, \lambda, \nu, q} .
$$


Proof. The following computation with generating functions shows the way:

$$
\begin{aligned}
& \sum_{\nu=0}^{\infty} \frac{(-t)^{\nu} \mathcal{F}_{\mathrm{JHC}, \lambda^{-1}, \nu, q}}{\{\nu\}_{q} !}=\frac{2}{\lambda^{-1} \mathrm{E}_{\frac{1}{q}}(-t)+1}=\frac{2 \lambda \mathrm{E}_{q}(t)}{\lambda \mathrm{E}_{q}(t)+1} \\
& =\lambda \sum_{\nu=0}^{\infty} \frac{t^{\nu} \mathcal{F}_{\mathrm{NWA}, \lambda, \nu, q}(1)}{\{\nu\}_{q} !} .
\end{aligned}
$$

Equating the coefficients of $t^{\nu}$ and using (70) gives (85).

Theorem 6.4. Assume that $g(t)$ in (67) and (80) are equal and even functions. Then

$$
\mathrm{f}_{\lambda^{-1}, \nu, q}^{(n)}(x)=(-1)^{\nu} \lambda^{n} \mathrm{e}_{\lambda, \nu, q}^{(n)}\left(\bar{n}_{q} \ominus_{q} x\right) .
$$

This implies a $q$-analogue of the complementary argument theorem $[9$, p. $634,(20)]$.

\section{Theorem 6.5.}

$$
\mathcal{F}_{\mathrm{JHC}, \lambda^{-1}, \nu, q}^{(n)}(x)=(-1)^{\nu} \lambda^{n} \mathcal{F}_{\mathrm{NWA}, \lambda, \nu, q}^{(n)}\left(\bar{n}_{q} \ominus_{q} x\right) .
$$

Theorem 6.6 (A q-analogue of $[6$, p. 8]).

$$
\begin{aligned}
& \sum_{k=0}^{\nu}\left(\begin{array}{l}
\nu \\
k
\end{array}\right)_{q} \mathcal{F}_{\mathrm{NWA}, \lambda, k, q}^{(2 n)}\left(x \ominus_{q} y\right)\left(\bar{n}_{q}\right)^{\nu-k} \\
& =\frac{1}{\lambda^{n}} \sum_{k=0}^{\nu}(-1)^{\nu-k}\left(\begin{array}{l}
\nu \\
k
\end{array}\right)_{q} \mathcal{F}_{\mathrm{NWA}, \lambda, k, q}^{(n)}(x) \mathcal{F}_{\mathrm{JHC}, \lambda-1, \nu-k, q}^{(n)}(y) .
\end{aligned}
$$

Proof. We have that

$$
\begin{aligned}
& \frac{2^{n}}{\left(\lambda \mathrm{E}_{q}(t)+1\right)^{n}} \mathrm{E}_{q}(x t) \frac{2^{n}}{\left(\lambda^{-1} \mathrm{E}_{\frac{1}{q}}(-t)+1\right)^{n}} \mathrm{E}_{q}(-y t) \\
& =\mathrm{E}_{q}\left(\left(x \ominus_{q} y\right) t\right) \frac{2^{n}}{\left(\lambda \mathrm{E}_{q}(t)+1\right)^{n}}\left(\frac{2 \lambda \mathrm{E}_{q}(t)}{\lambda \mathrm{E}_{q}(t)+1}\right)^{n},
\end{aligned}
$$

which implies that

$$
\begin{aligned}
& \sum_{\nu=0}^{\infty} \frac{t^{\nu} \mathcal{F}_{\mathrm{NWA}, \lambda, \nu, q}^{(2 n)}\left(x \ominus_{q} y\right)}{\{\nu\}_{q} !} \sum_{l=0}^{\infty} \frac{\left(\bar{n}_{q} t\right)^{l}}{\{l\}_{q} !} \\
& =\frac{1}{\lambda^{n}} \sum_{\nu=0}^{\infty} \frac{t^{\nu} \mathcal{F}_{\mathrm{NWA}, \lambda, \nu, q}^{(n)}(x)}{\{\nu\}_{q} !} \sum_{m=0}^{\infty} \frac{(-t)^{m} \mathcal{F}_{\mathrm{JHC}, \lambda-1, m, q}^{(n)}(y)}{\{m\}_{q} !} .
\end{aligned}
$$

Formula (89) now follows on equating the coefficients of $t^{\nu}$. 
7. More on $q$-Apostol-Bernoulli and Euler polynomials. It turns out, by simple umbral manipulation that many of the formulas in [3, Section 4.3] are also valid for $q$-Apostol-Bernoulli and Euler polynomials, and these equations which we now present will also be new for the case $q=1$. We first define these polynomials of negative order.

Definition 22. The generalized NWA $q$-Apostol-Bernoulli polynomials of negative order $-n$ are given by

$$
\mathcal{B}_{\mathrm{NWA}, \lambda, \nu, q}^{(-n)}(x) \equiv \frac{\{\nu\}_{q} !}{\{\nu+n\}_{q} !} \triangle_{\mathrm{NWA}, \mathcal{A}, q}^{n} x^{\nu+n} .
$$

The generalized NWA $q$-Apostol-Euler polynomials of negative order $-n$ are given by

$$
\mathcal{F}_{\mathrm{NWA}, \lambda, \nu, q}^{(-n)}(x) \equiv \nabla_{\mathrm{NWA}, \mathcal{A}, q}^{n} x^{\nu} .
$$

Theorem 7.1. A generalization of [3, 4.249]:

$$
\mathcal{B}_{\mathrm{NWA}, \lambda, \nu, q}^{(-n-p)}\left(x \oplus_{q} y\right) \ddot{=}\left(\mathcal{B}_{\mathrm{NWA}, \lambda, q}^{(-n)}(x) \oplus_{q} \mathcal{B}_{\mathrm{NWA}, \lambda, q}^{(-p)}(y)\right)^{\nu},
$$

and the same for NWA q-Apostol-Euler polynomials

A special case is the following formula:

$$
\mathcal{B}_{\mathrm{NWA}, \lambda, \nu, q}^{(-n)}\left(x \oplus_{q} y\right) \stackrel{=}{=}\left(\mathcal{B}_{\mathrm{NWA}, \lambda, q}^{(-n)}(x) \oplus_{q} y\right)^{\nu},
$$

and the same for NWA q-Apostol-Euler polynomials.

Theorem 7.2. A generalization of [3, 4.251, 4.252]:

If $n, p \in \mathbb{Z}$ then

$$
\mathcal{B}_{\mathrm{NWA}, \lambda, \nu, q}^{(n+p)} \ddot{=}\left(\mathcal{B}_{\mathrm{NWA}, \lambda, q}^{(n)} \oplus_{q} \mathcal{B}_{\mathrm{NWA}, \lambda, q}^{(p)}\right)^{\nu},
$$

and the same for NWA q-Apostol-Euler numbers.

Theorem 7.3. A generalization of [3, 4.253]:

$$
\left(x \oplus_{q} y\right)^{\nu} \ddot{=}\left(\mathcal{B}_{\mathrm{NWA}, \lambda, q}^{(-n)}(x) \oplus_{q} \mathcal{B}_{\mathrm{NWA}, \lambda, q}^{(n)}(y)\right)^{\nu},
$$

A generalization of $[3,4.254]$ :

$$
\left(x \oplus_{q} y\right)^{\nu} \ddot{=}\left(\mathcal{F}_{\mathrm{NWA}, \lambda, q}^{(-n)}(x) \oplus_{q} \mathcal{F}_{\mathrm{NWA}, \lambda, q}^{(n)}(y)\right)^{\nu} .
$$

Proof. Put $p=-n$ in (94).

In particular for $y=0$, we obtain

$$
\begin{aligned}
& x^{\nu}=\left(\mathcal{B}_{\mathrm{NWA}, \lambda, q}^{(-n)} \oplus_{q} \mathcal{B}_{\mathrm{NWA}, \lambda, q}^{(n)}(x)\right)^{\nu}, \\
& x^{\nu} \ddot{=}\left(\mathcal{F}_{\mathrm{NWA}, \lambda, q}^{(-n)} \oplus_{q} \mathcal{F}_{\mathrm{NWA}, \lambda, q}^{(n)}(x)\right)^{\nu} .
\end{aligned}
$$


This can be restated in the form

$$
\begin{aligned}
& x^{\nu}=\sum_{s=0}^{\nu} \frac{\mathcal{B}_{\mathrm{NWA}, \lambda, s, q}^{(-n)}}{\{s\}_{q} !} \mathrm{D}_{q}^{s} \mathcal{B}_{\mathrm{NWA}, \lambda, \nu, q}^{(n)}(x), \\
& x^{\nu}=\sum_{s=0}^{\nu} \frac{\mathcal{F}_{\mathrm{NWA}, \lambda, s, q}^{(-n)}}{\{s\}_{q} !} \mathrm{D}_{q}^{s} \mathcal{F}_{\mathrm{NWA}, \lambda, \nu, q}^{(n)}(x) .
\end{aligned}
$$

We conclude that the NWA $q$-Apostol-Bernoulli and NWA $q$-Apostol-Euler polynomials satisfy linear $q$-difference equations with constant coefficients.

The following theorem is useful for the computation of NWA $q$-ApostolBernoulli and NWA $q$-Apostol-Euler polynomials of positive order. This is because the polynomials of negative order are of simpler nature and can easily be computed. When the $\mathrm{B}_{\mathrm{NWA}, \lambda, s, q}^{(-n)}$ etc. are known, (103) can be used to compute the $\mathrm{B}_{\mathrm{NWA}, \lambda, s, q}^{(n)}$.

Theorem 7.4. A generalization of [3, 4.259]:

$$
\sum_{s=0}^{\nu}\left(\begin{array}{l}
\nu \\
s
\end{array}\right)_{q} \mathcal{B}_{\mathrm{NWA}, \lambda, s, q}^{(n)} \mathcal{B}_{\mathrm{NWA}, \lambda, \nu-s, q}^{(-n)}=\delta_{\nu, 0} .
$$

A generalization of $[3,4.260]$ :

$$
\sum_{s=0}^{\nu}\left(\begin{array}{l}
\nu \\
s
\end{array}\right)_{q} \mathcal{F}_{\mathrm{NWA}, \lambda, s, q}^{(n)} \mathcal{F}_{\mathrm{NWA}, \lambda, \nu-s, q}^{(-n)}=\delta_{\nu, 0} .
$$

Proof. Put $x=y=0$ in (97) and (98).

Theorem 7.5 (A generalization of [3, 4.261]). Under the assumption that $f(x)$ is analytic with $q$-Taylor expansion

$$
f(x)=\sum_{\nu=0}^{\infty} \mathrm{D}_{q}^{\nu} f(0) \frac{x^{\nu}}{\{\nu\}_{q} !},
$$

we can express powers of $\triangle_{\mathrm{NWA}, \mathcal{A}, q}$ and $\nabla_{\mathrm{NWA}, \mathcal{A}, q}$ operating on $f(x)$ as powers of $\mathrm{D}_{q}$ as follows. These series converge when the absolute value of $x$ is small enough:

$$
\begin{gathered}
\triangle_{\mathrm{NWA}, \mathcal{A}, q}^{n} f(x)=\sum_{\nu=0}^{\infty} \mathrm{D}_{q}^{\nu+n} f(0) \frac{\mathcal{B}_{\mathrm{NWA}, \lambda, \nu, q}^{(-n)}(x)}{\{\nu\}_{q} !} \\
\nabla_{\mathrm{NWA}, \mathcal{A}, q}^{n} f(x)=\sum_{\nu=0}^{\infty} \mathrm{D}_{q}^{\nu} f(0) \frac{\mathcal{F}_{\mathrm{NWA}, \lambda, \nu, q}^{(-n)}(x)}{\{\nu\}_{q} !}
\end{gathered}
$$

Proof. Use formulas (45) and (54). 
Putting $f(x)=\mathrm{E}_{q}(x t)$ we obtain the generating function of the NWA $q$ Apostol-Bernoulli and NWA $q$-Apostol-Euler polynomials of negative order:

$$
\begin{aligned}
& \frac{\left(\lambda \mathrm{E}_{q}(t)-1\right)^{n}}{t^{n}} \mathrm{E}_{q}(x t)=\sum_{\nu=0}^{\infty} \frac{t^{\nu}}{\{\nu\}_{q} !} \mathcal{B}_{\mathrm{NWA}, \lambda, \nu, q}^{(-n)}(x), \\
& \frac{\left(\lambda \mathrm{E}_{q}(t)+1\right)^{n}}{2^{n}} \mathrm{E}_{q}(x t)=\sum_{\nu=0}^{\infty} \frac{t^{\nu}}{\{\nu\}_{q} !} \mathcal{F}_{\mathrm{NWA}, \lambda, \nu, q}^{(-n)}(x) .
\end{aligned}
$$

Theorem 7.6 (A generalization of $[3,4.268]$ ).

$$
\sum_{k=0}^{\infty} \frac{\mathcal{B}_{\mathrm{NWA}, \lambda, k, q}^{(n)}(x)}{\{k\}_{q} !} \triangle_{\mathrm{NWA}, \mathcal{A}, q}^{n} \mathrm{D}_{q}^{k} f(y)=\mathrm{D}_{q, x}^{n} f\left(x \oplus_{q} y\right) .
$$

Proof. Replace $f(x)$ by $f\left(x \oplus_{q} y\right)$ in (45):

$$
\begin{aligned}
\lambda f( & \left.\mathcal{B}_{\mathrm{NWA}, \lambda, q}^{(n)}(x) \oplus_{q} y \oplus_{q} 1\right)-f\left(\mathcal{B}_{\mathrm{NWA}, \lambda, q}^{(n)}(x) \oplus_{q} y\right) \\
& =\mathrm{D}_{q} f\left(\mathrm{~B}_{\mathrm{NWA}, \lambda, q}^{(n-1)}(x) \oplus_{q} y\right) .
\end{aligned}
$$

Use the umbral formula $[3,4.21]$ to get

$$
\sum_{k=0}^{\infty} \frac{\mathcal{B}_{\mathrm{NWA}, \lambda, k, q}^{(n)}(x)}{\{k\}_{q} !} \triangle_{\mathrm{NWA}, \mathcal{A}, q} \mathrm{D}_{q}^{k} f(y)=\sum_{k=0}^{\infty} \frac{\mathcal{B}_{\mathrm{NWA}, k, q}^{(n-1)}(x)}{\{k\}_{q} !} \mathrm{D}_{q}^{k+1} f(y) .
$$

Apply the operator $\triangle_{\mathrm{NWA}, \mathcal{A}, q}^{n-1}$ with respect to $y$ to both sides and use (106):

$$
\begin{aligned}
& \sum_{k=0}^{\infty} \frac{\mathcal{B}_{\mathrm{NWA}, \lambda, k, q}^{(n)}(x)}{\{k\}_{q} !} \triangle_{\mathrm{NWA}, \mathcal{A}, q}^{n} \mathrm{D}_{q}^{k} f(y) \\
& \quad=\sum_{k=0}^{\infty} \frac{\mathcal{B}_{\mathrm{NWA}, \lambda, k, q}^{(n-1)}(x)}{\{k\}_{q} !} \sum_{l=0}^{\infty} \mathrm{D}_{q}^{k+l+n} f(0) \frac{\mathcal{B}_{\mathrm{NWA}, \lambda, l, q}^{(-n+1)}(y)}{\{l\}_{q} !} .
\end{aligned}
$$

Finally use $[3,4.21],[3,4.34],(97)$ to rewrite the righthand side.

Corollary 7.7 (A generalization of $[3,4.272]$ ). Let $\varphi(x)$ be a polynomial of degree $\nu$. A solution $f(x)$ of the q-difference equation

$$
\triangle_{\mathrm{NWA}, \mathcal{A}, q}^{n} f(x)=\mathrm{D}_{q}^{n} \varphi(x)
$$

is given by

$$
f\left(x \oplus_{q} y\right)=\sum_{k=0}^{\nu} \frac{\mathcal{B}_{\mathrm{NWA}, \lambda, k, q}^{(n)}(x)}{\{k\}_{q} !} \mathrm{D}_{q}^{k} \varphi(y)
$$


Proof. The LHS of (115) can be written as $\varphi\left(\mathcal{B}_{\mathrm{NWA}, \lambda, q}^{(n)}(x) \oplus_{q} y\right)$, because if we apply $\triangle_{\mathrm{NWA}, q, x}^{n}$ to both sides we get

$$
\begin{aligned}
& \triangle_{\mathrm{NWA}, \mathcal{A}, q}^{n} f\left(x \oplus_{q} y\right)=\mathrm{D}_{q, x}^{n} \varphi\left(x \oplus_{q} y\right) \\
& =\triangle_{\mathrm{NWA}, \mathcal{A}, q}^{n} \varphi\left(\mathcal{B}_{\mathrm{NWA}, \lambda, q}^{(n)}(x) \oplus_{q} y\right) .
\end{aligned}
$$

Theorem 7.8 (A generalization of [3, 4.275]).

$$
\sum_{k=0}^{\infty} \frac{\mathcal{F}_{\mathrm{NWA}, \lambda, k, q}^{(n)}(x)}{\{k\}_{q} !} \nabla_{\mathrm{NWA}, \mathcal{A}, q}^{n} \mathrm{D}_{q}^{k} f(y)=f\left(x \oplus_{q} y\right)
$$

Proof. Replace $f(x)$ by $f\left(x \oplus_{q} y\right)$ in (69):

$$
\begin{aligned}
& \frac{1}{2}\left(\lambda f\left(\mathcal{F}_{\mathrm{NWA}, \lambda, q}^{(n)}(x) \oplus_{q} y \oplus_{q} 1\right)+f\left(\mathcal{F}_{\mathrm{NWA}, \lambda, q}^{(n)}(x) \oplus_{q} y\right)\right) \\
& \quad=f\left(\mathcal{F}_{\mathrm{NWA}, \lambda, q}^{(n-1)}(x) \oplus_{q} y\right) .
\end{aligned}
$$

Use the umbral formula $[3,4.21]$ to get

$$
\sum_{k=0}^{\infty} \frac{\mathcal{F}_{\mathrm{NWA}, \lambda, k, q}^{(n)}(x)}{\{k\}_{q} !} \nabla_{\mathrm{NWA}, \mathcal{A}, q} \mathrm{D}_{q}^{k} f(y)=\sum_{k=0}^{\infty} \frac{\mathcal{F}_{\mathrm{NWA}, \lambda, k, q}^{(n-1)}(x)}{\{k\}_{q} !} \mathrm{D}_{q}^{k} f(y)
$$

Apply the operator $\nabla_{\mathrm{NWA}, \mathcal{A}, q}^{n-1}$ with respect to $y$ to both sides and use (107):

$$
\begin{aligned}
& \sum_{k=0}^{\infty} \frac{\mathcal{F}_{\mathrm{NWA}, \lambda, k, q}^{(n)}(x)}{\{k\}_{q} !} \nabla_{\mathrm{NWA}, \mathcal{A}, q}^{n} \mathrm{D}_{q}^{k} f(y) \\
& =\sum_{k=0}^{\infty} \frac{\mathcal{F}_{\mathrm{NWA}, \lambda, k, q}^{(n-1)}(x)}{\{k\}_{q} !} \sum_{l=0}^{\infty} \mathrm{D}_{q}^{k+l} f(0) \frac{\mathcal{F}_{\mathrm{NWA}, \lambda, l, q}^{(-n+1)}(y)}{\{l\}_{q} !} .
\end{aligned}
$$

Finally use [3, 4.21], [3, 4.34], (98) to rewrite the righthand side.

Corollary 7.9 (A generalization of $[3,4.279]$ ). Let $\varphi(x)$ be a polynomial of degree $\nu$. A solution $f(x)$ of the q-difference equation

$$
\nabla_{\mathrm{NWA}, \mathcal{A}, q}^{n} f(x)=\varphi(x)
$$

is given by

$$
f\left(x \oplus_{q} y\right)=\sum_{k=0}^{\nu} \frac{\mathcal{F}_{\mathrm{NWA}, \lambda, k, q}^{(n)}(x)}{\{k\}_{q} !} \mathrm{D}_{q}^{k} \varphi(y) .
$$

Proof. The LHS of (122) can be written as $\varphi\left(\mathcal{F}_{\mathrm{NWA}, \lambda, q}^{(n)}(x) \oplus_{q} y\right)$, because if we apply $\nabla_{\mathrm{NWA}, \mathcal{A}, q, x}^{n}$ to both sides we get

$$
\nabla_{\mathrm{NWA}, \mathcal{A}, q}^{n} f\left(x \oplus_{q} y\right)=\varphi\left(x \oplus_{q} y\right)=\nabla_{\mathrm{NWA}, \mathcal{A}, q}^{n} \varphi\left(\mathcal{F}_{\mathrm{NWA}, \lambda, q}^{(n)}(x) \oplus_{q} y\right) .
$$


8. Multiplicative $\boldsymbol{q}$-Appell polynomials. In this section we very briefly discuss multiplicative $q$-Appell polynomials with $f_{n}(t)$ equal to $h(t)^{n}$. It turns out, by simple umbral manipulation that many of the formulas in [3, Section 4.3] are also valid for multiplicative $q$-Appell polynomials, and these equations will be presented in another article. Throughout, we denote the multiplicative $q$-Appell polynomials by $\Phi_{\mathcal{N}, \nu, q}^{(n)}(x)$.

Definition 23. Under the assumption that the function $h(t)^{n}$ can be expressed analytically in $\mathbb{R}[[t]]$, and for $f_{n}(t)$ of the form $h(t)^{n}$, we call the $q$-Appell polynomial $\Phi_{q}$ in (14) multiplicative.

$$
\mathrm{E}_{q}(x t) h(t)^{n}=\sum_{\nu=0}^{\infty} \frac{t^{\nu}}{\{\nu\}_{q} !} \Phi_{\mathcal{\mathcal { N }}, \nu, q}^{(n)}(x), n \in \mathbb{Z} .
$$

Then we have

Theorem 8.1. If $\sum_{l=1}^{s} n_{l}=n, n \in \mathbb{N}$,

$$
\Phi_{\mathcal{M}, k, q}^{(n)}\left(x_{1} \oplus_{q} \ldots \oplus_{q} x_{s}\right)=\sum_{m_{1}+\ldots+m_{s}=k}\left(\begin{array}{c}
k \\
m_{1}, \ldots, m_{s}
\end{array}\right) \prod_{q}^{s} \Phi_{j=1}^{\left(n_{j}\right)}\left(x_{j}\right),
$$

where we assume that $n_{j}$ operates on $x_{j}$.

Proof. Compare with [3, 4.242]: In umbral notation we have, like in the classical case:

$$
\left(x_{1} \oplus_{q} \ldots \oplus_{q} x_{s} \oplus_{q} \bar{n}_{q} \gamma\right)^{k} \sim\left(\left(x_{1} \oplus_{q} \overline{n_{1}} \gamma^{\prime}\right) \oplus_{q} \ldots \oplus_{q}\left(x_{s} \oplus_{q} \bar{n}_{s} \gamma^{\prime \prime}\right)\right)^{k}
$$

where $\gamma^{\prime}, \ldots, \gamma^{\prime \prime}$ are distinct umbrae, each equivalent to $\gamma$.

9. The $q$-logarithm. We wish to investigate the existence of a real inverse for $\mathrm{E}_{q}(x)$.

Theorem 9.1. The function $\mathrm{E}_{q}(x),-\infty<x<(1-q)^{-1}$ has an inverse.

Proof. It suffices to show that the logarithmic derivative of $\mathrm{E}_{q}(x)$ is $>0$. However, this follows from

$$
\mathrm{D}\left(\log \mathrm{E}_{q}(x)\right)=\sum_{m=0}^{\infty} \frac{(1-q) q^{m}}{1-x(1-q) q^{m}}>0,0<q<1 .
$$

Definition 24. The $q$-logarithm $\log _{q}(x)$ is the inverse function of $\mathrm{E}_{q}(x)$, $-\infty<x<(1-q)^{-1}, 0<q<1$.

Theorem 9.2. The $q$-logarithm $\log _{q}(x)$ has the following properties ( $x$ and $y$ have small real values, $n \in \mathbb{N}$ ):

(1) Its domain is $\left.\mathbb{R}_{+}=\right] 0, \infty[$, and its range is $]-\infty,(1-q)^{-1}[$.

(2) It is strictly increasing. 
(3) $\log _{q}(x) \oplus_{q} \log _{q}(y) \cong \log _{q}(x y)$.

(4) $\log _{q}(x) \oplus_{q} \log _{q}(x) \oplus_{q} \cdots \oplus_{q} \log _{q}(x) \cong \log _{q}\left(x^{n}\right)$.

Theorem 9.3 (A q-analogue of [8, p. 293 (13)]).

$$
\mathcal{F}_{\lambda, \nu, q}^{(n)}(x)=\mathrm{E}_{\frac{1}{q}}\left(-x \log _{q}(\lambda)\right) \sum_{k=0}^{\infty} \mathrm{F}_{\mathrm{NWA}, k+\nu, q}^{(n)}(x) \frac{\left(\log _{q}(\lambda)\right)^{k}}{\{k\}_{q} !} .
$$

Proof. We equate the generating functions in the following way:

$$
\begin{aligned}
& \sum_{\nu=0}^{\infty} \frac{t^{\nu} \mathcal{F}_{\lambda, \nu, q}^{(n)}(x)}{\{\nu\}_{q} !} \\
& =\mathrm{E}_{\frac{1}{q}}\left(-x \log _{q}(\lambda)\right) \frac{2^{n}}{\left(\mathrm{E}_{q}\left(t \oplus_{q} \log _{q}(\lambda)\right)+1\right)^{n}} \mathrm{E}_{q}\left(x\left(t \oplus_{q} \log _{q}(\lambda)\right)\right) \\
& =\mathrm{E}_{\frac{1}{q}}\left(-x \log _{q}(\lambda)\right) \sum_{k=0}^{\infty} \mathrm{F}_{\mathrm{NWA}, k, q}^{(n)}(x) \frac{\left(t \oplus_{q} \log _{q}(\lambda)\right)^{k}}{\{k\}_{q} !} \\
& =\mathrm{E}_{\frac{1}{q}}\left(-x \log _{q}(\lambda)\right) \sum_{k=0}^{\infty} \mathrm{F}_{\mathrm{NWA}, k, q}^{(n)}(x) \sum_{m=0}^{k} \frac{t^{m}\left(\log _{q}(\lambda)\right)^{k-m}}{\{k-m\}_{q} !\{m\}_{q} !} \\
& =\mathrm{E}_{\frac{1}{q}}\left(-x \log _{q}(\lambda)\right) \sum_{m=0}^{\infty} \frac{t^{m}}{\{m\}_{q} !} \sum_{k=0}^{\infty} \mathrm{F}_{\mathrm{NWA}, k+m, q}^{(n)}(x) \frac{\left(\log _{q}(\lambda)\right)^{k}}{\{k\}_{q} !}
\end{aligned}
$$

10. Appendix: multiple $q$-Appell polynomials. Of course there are many ways to define multiple $q$-Appell polynomials; in this paper we concentrate on one of the simplest approaches in the spirit of Lee.

Definition 25 (A q-analogue of [7, p. 2135]). For every power series

$$
f\left(t_{1}, t_{2}\right) \equiv \sum_{k, l=0}^{\infty} a_{k, l} \frac{t_{1}^{k} t_{2}^{l}}{\{k\}_{q} !\{l\}_{q} !}
$$

with $f(0,0) \neq 0$, the multiple $q$-Appell polynomials $\Phi_{k, l ; q}(x)$ of degree $k, l$ have the following generating function:

$$
f\left(t_{1}, t_{2}\right) \mathrm{E}_{q}\left(x\left(t_{1} \oplus_{q} t_{2}\right)\right)=\sum_{k, l=0}^{\infty} \frac{t_{1}^{k} t_{2}^{l}}{\{k\}_{q} !\{l\}_{q} !} \Phi_{k, l ; q}(x) .
$$

Theorem 10.1 (A q-analogue of [7, p. 2135]). Let $\left\{\Phi_{k, l ; q}(x)\right\}_{k, l=0}^{\infty}$ be a multiple q-Appell polynomial. Then we have equivalently:

(1) $\left\{\Phi_{k, l ; q}(x)\right\}_{k, l=0}^{\infty}$ is a set of multiple $q$-Appell polynomials. 
(2) There exists a sequence $\left\{\Phi_{k, l ; q}\right\}_{k, l=0}^{\infty}$ with $\Phi_{0,0 ; q} \neq 0$ such that

$$
\Phi_{k, l ; q}(x)=\sum_{k_{1}=0}^{k} \sum_{l_{1}=0}^{l}\left(\begin{array}{c}
k \\
k_{1}
\end{array}\right)_{q}\left(\begin{array}{l}
l \\
l_{1}
\end{array}\right)_{q} \Phi_{k-k_{1}, l-l_{1}, q} x^{k_{1}+l_{1}} .
$$

(3) Let

$$
\Phi_{k, l ; \alpha, \beta ; q}(x)=\sum_{k_{1}=0}^{k} \sum_{l_{1}=0}^{l}\left(\begin{array}{c}
k \\
k_{1}
\end{array}\right)_{q}\left(\begin{array}{l}
l \\
l_{1}
\end{array}\right)_{q} \Phi_{k-k_{1}, l-l_{1}, q} x^{k_{1}+l_{1}} q^{\alpha k_{1}+\beta l_{1}} .
$$

Then for each $n_{1}+n_{2} \geq 1$, we have

$$
\mathrm{D}_{q} \Phi_{k, l ; q}(x)=\{k\}_{q} \Phi_{k-1, l ; 0,1 ; q}(x)+\{l\}_{q} \Phi_{k, l-1 ; q}(x) .
$$

(4) There exists a sequence $\left\{\Phi_{k, l ; q}\right\}_{k, l=0}^{\infty}$ with $\Phi_{0,0 ; q} \neq 0$ such that

$$
\begin{aligned}
& \Phi_{k, l ; q}(x) \\
& =\sum_{k_{1}=0}^{k} \sum_{l_{1}=0}^{l}\left(\begin{array}{c}
k \\
k_{1}
\end{array}\right)_{q}\left(\begin{array}{l}
l \\
l_{1}
\end{array}\right)_{q} \frac{\left\{k+l-k_{1}-l_{1}\right\}_{q} !}{\{k+l\}_{q} !} \Phi_{k_{1}, l_{1} ; q} \mathrm{D}_{q}^{k_{1}+l_{1}} x^{k+l} .
\end{aligned}
$$

Proof. $(1) \Rightarrow(2)$. This follows since each $t_{1}$ and $t_{2}$ provides a generating function for a $q$-Appell polynomial.

$(2) \Rightarrow(3)$. By the identity

$$
\begin{aligned}
& \left\{k_{1}+k_{2}\right\}_{q}\left(\begin{array}{l}
n_{1} \\
k_{1}
\end{array}\right)_{q}\left(\begin{array}{l}
n_{2} \\
k_{2}
\end{array}\right)_{q} \\
& \quad=q^{k_{2}}\left\{n_{1}\right\}_{q}\left(\begin{array}{l}
n_{1}-1 \\
k_{1}-1
\end{array}\right)_{q}\left(\begin{array}{l}
n_{2} \\
k_{2}
\end{array}\right)_{q}+\left\{n_{2}\right\}_{q}\left(\begin{array}{l}
n_{1} \\
k_{1}
\end{array}\right)_{q}\left(\begin{array}{l}
n_{2}-1 \\
k_{2}-1
\end{array}\right)_{q}, \\
& k_{1} \geq 1, k_{2} \geq 1,
\end{aligned}
$$

we have

$$
\begin{aligned}
& \mathrm{D}_{q} \Phi_{k, l ; q}(x)=\sum_{k_{1}=0}^{k} \sum_{l_{1}=0}^{l}\left\{k_{1}+l_{1}\right\}_{q}\left(\begin{array}{c}
k \\
k_{1}
\end{array}\right)_{q}\left(\begin{array}{l}
l \\
l_{1}
\end{array}\right)_{q} \Phi_{k-k_{1}, l-l_{1}, q} x^{k_{1}+l_{1}-1} \\
& \stackrel{\operatorname{by}(136)}{=} \sum_{k_{1}=0}^{k} \sum_{l_{1}=0}^{l}\left(q^{l_{1}}\{k\}_{q}\left(\begin{array}{c}
k-1 \\
k_{1}-1
\end{array}\right)_{q}\left(\begin{array}{l}
l \\
l_{1}
\end{array}\right)_{q}\right. \\
& \left.+\{l\}_{q}\left(\begin{array}{c}
k \\
k_{1}
\end{array}\right)_{q}\left(\begin{array}{c}
l-1 \\
l_{1}-1
\end{array}\right)_{q}\right) \Phi_{k-k_{1}, l-l_{1}, q} x^{k_{1}+l_{1}-1} \\
& =\{k\}_{q} \sum_{k_{1}=0}^{k-1} \sum_{l_{1}=0}^{l}\left(\begin{array}{c}
k-1 \\
k_{1}
\end{array}\right)_{q}\left(\begin{array}{l}
l \\
l_{1}
\end{array}\right)_{q} \Phi_{k-1-k_{1}, l-l_{1}, q} x^{k_{1}+l_{1}} q^{l_{1}} \\
& +\{l\}_{q} \sum_{k_{1}=0}^{k} \sum_{l_{1}=0}^{l-1}\left(\begin{array}{c}
k \\
k_{1}
\end{array}\right)_{q}\left(\begin{array}{c}
l-1 \\
l_{1}
\end{array}\right)_{q} \Phi_{k-k_{1}, l-1-l_{1}, q} x^{k_{1}+l_{1}}=\text { RHS. }
\end{aligned}
$$


The last statement follows after performing the $q$-derivation and changing summation indices.

11. Discussion. We have discussed six new types of $q$-Appell polynomials, and a multiple form, but the trend is clear; there are many advantages with this general kind of function. This theory is very flexible and ready for generalizations. In another paper we will prove that the $q$-Appell polynomials (and the Appell polynomials as well) form a commutative ring. We hope that this article will advertise $q$-Appell polynomials, which are not very well known; as a coincidence we mention that Paul Appell (1855-1930) was mathematics teacher of Marie Curie in Paris, they became a very successful team.

\section{REFERENCES}

[1] Apostol, T. M., On the Lerch zeta function, Pacific J. Math. 1 (1951), 161-167.

[2] Dere, R., Simsek, Y., Srivastava, H. M., A unified presentation of three families of generalized Apostol type polynomials based upon the theory of the umbral calculus and the umbral algebra, J. Number Theory 133, no. 10 (2013), 3245-3263.

[3] Ernst, T., A comprehensive treatment of q-calculus, Birkhäuser, Basel, 2012.

[4] Ernst, T., q-Pascal and $q$-Wronskian matrices with implications to $q$-Appell polynomials, J. Discrete Math. 2013.

[5] Jordan, Ch., Calculus of finite differences, Third Edition, Chelsea Publishing Co., New York, 1950.

[6] Kim M., Hu S., A note on the Apostol-Bernoulli and Apostol-Euler polynomials, Publ. Math. Debrecen 5587 (2013), 1-16.

[7] Lee, D. W., On multiple Appell polynomials, Proc. Amer. Math. Soc. 139, no. 6 (2011), 2133-2141.

[8] Luo, Q.-M., Srivastava, H. M., Some generalizations of the Apostol-Bernoulli and Apostol-Euler polynomials, J. Math. Anal. Appl. 308, no. 1 (2005), 290-302.

[9] Luo, Q.-M., Srivastava, H. M., Some relationships between the Apostol-Bernoulli and Apostol-Euler polynomials, Comput. Math. Appl. 51, no. 3-4 (2006), 631-642.

[10] Luo, Q.-M., Apostol-Euler polynomials of higher order and Gaussian hypergeometric functions, Taiwanese J. Math. 10, no. 4 (2006), 917-925.

[11] Milne-Thomson, L. M., The Calculus of Finite Differences, Macmillan and Co., Ltd., London, 1951.

[12] Nørlund, N. E., Differenzenrechnung, Springer-Verlag, Berlin, 1924.

[13] Pintér, Á, Srivastava, H. M., Addition theorems for the Appell polynomials and the associated classes of polynomial expansions, Aequationes Math. 85, no. 3 (2013), 483-495.

[14] Sandor, J., Crstici, B., Handbook of number theory II, Kluwer Academic Publishers, Dordrecht, 2004.

[15] Srivastava, H. M., Özarslan, M. A., Kaanoglu, C., Some generalized Lagrange-based Apostol-Bernoulli, Apostol-Euler and Apostol-Genocchi polynomials, Russ. J. Math. Phys. 20, no. 1 (2013), 110-120.

[16] Wang, W., Wang, W., Some results on power sums and Apostol-type polynomials, Integral Transforms Spec. Funct. 21, no. 3-4 (2010), 307-318.

[17] Ward, M., A calculus of sequences, Amer. J. Math. 58 (1936), 255-266. 
Thomas Ernst

Department of Mathematics

Uppsala University

P.O. Box 480, SE-751 06 Uppsala

Sweden

e-mail: thomas@math.uu.se

Received February 1, 2014 